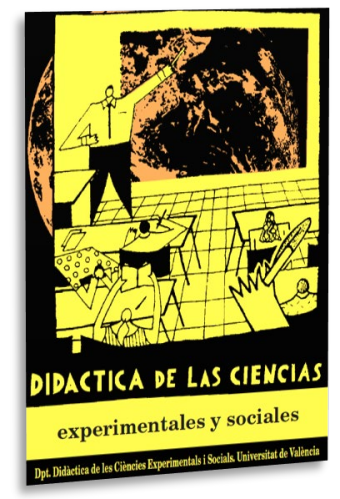

\title{
Pensar en Clío: la evolución de las concepciones historiográficas del alumnado de 1 o de Educación Secundaria Obligatoria
}

\author{
Thinking of Clio: the evolution of the \\ historiographic conceptions of students of the \\ first year of compulsory Secondary Education
}

DOI: $10.7203 / D C E S .39 .15512$

\author{
Francisco J. Fraile-Delgado \\ IES Puerta del Andévalo (S. Bartolomé de la Torre, Huelva), franciscojfraile@hotmail.com \\ ORCID iD: https://orcid.org/0000-0003-2885-6639
}

\begin{abstract}
RESUMEN: En este trabajo se ha optado por aplicar una metodología activa para trabajar los contenidos y competencias asociadas al pensamiento histórico. El objetivo de esta investigación es comprobar cómo evolucionan las concepciones historiográficas del alumnado de $1^{\circ}$ de Educación Secundaria Obligatoria (ESO). Para ello, se ha optado por una estrategia plurimetodológica. En un primer momento se realizó un estudio descriptivo que permitiese comprobar la evolución de las ideas del alumnado y, posteriormente, un análisis de casos múltiple, para comprender qué aspectos han incidido en esa evolución. La muestra la componían un grupo de 23 alumnos, a los que se les ha pasado un cuestionario inicial y final. Se seleccionaron 3 estudiantes para el análisis de casos, a los que se les ha realizado una entrevista semiestructurada. El análisis y los resultados revelan una evolución significativa de las ideas historiográficas del alumnado, teniendo el desarrollo del pensamiento histórico como principal referencia del proceso de enseñanza-aprendizaje.
\end{abstract}

Palabras Clave: pensamiento crítico, historia, Ciencias Sociales, enseñanza secundaria, didáctica

\begin{abstract}
In this work, we have chosen to apply an active methodology to work on the contents and competences associated with historical thought. The aim of this research is to check how the historiographic conceptions of students in the first year of compulsory Secondary Education (ESO by its Spanish acronym), aged 11-12 years old, evolve. To do so, a multi-methodological strategy has been chosen. Firstly, a descriptive study was carried out to check the evolution of the students' ideas and, later, a multiple case analysis was made to understand what aspects had influenced this evolution. The sample was a group of 23 students, who were given an initial and final questionnaire. Subsequently, three students were selected for the case analysis and underwent a semi-structured interview. The analysis and the results reveal a significant evolution of the students' historiographic ideas, having the development of historical thought as the main reference of the teaching-learning process.
\end{abstract}

KEYWORDS: critical sense, history, Social Sciences, Secondary Education, didactics

Fecha de recepción: julio de 2019

Fecha de aceptación: febrero de 2020 


\section{INTRODUCCIÓN}

Las investigaciones sobre la didáctica de la historia y de las ciencias sociales son numerosas. No obstante, y a pesar de las novedosas iniciativas que han tenido lugar, parece que en las aulas aún siguen imperando las prácticas más tradicionales. Por otra parte, las concepciones del alumnado también han sido objeto de estudio por la investigación educativa (Fuentes, 2002). En concreto, en este artículo nos interesamos por las concepciones del alumnado sobre el conocimiento histórico, las cuales, dentro del aula, están influidas por la forma en la que se trabaje la materia. Así pues, el enfoque positivista de la historia puede suponer que el alumnado acabe por tener una imagen de la disciplina histórica que queda reducida a la memorización de los hechos y de las fechas más relevantes, esto es, reduciendo el pensamiento histórico a una visión enciclopédica (Gómez Carrasco y Miralles Martínez, 2017).

Es por ello que en el siguiente estudio se pretende, por una parte, plantear una secuencia metodológica que busque el desarrollo de un pensamiento histórico más complejo y un papel más activo del alumnado en el proceso de enseñanza aprendizaje. Por otra parte, se pretende comprobar cómo evolucionan las concepciones historiográficas del alumnado siguiendo este enfoque más centrado en la adquisición de las diferentes competencias asociadas al pensamiento histórico. Para ello, ha sido fundamental los postulados teóricos asociados a la enseñanza del pensamiento histórico, así como las aportaciones de Concha Fuentes sobre las concepciones del alumnado sobre la historia, especialmente en lo referido a la metodología, puesto que ha sido un marco de referencia para el desarrollo de esta investigación.

\section{LA CLASE DE HISTORIA}

La didáctica de la historia y las investigaciones educativas se han centrado en el estudio de lo que ocurre en la clase de historia. En este sentido, cabe destacar que en la actualidad siguen teniendo vigencia los enfoques más tradicionales para enseñar historia, conviviendo con nuevas pautas innovadoras. Claro está que las instituciones educativas se muestran muy resistentes al cambio (Merchán Iglesias, 2005) y, de hecho, López Facal afirma que:
A la hora de explicar tanto los cambios como las permanencias o rutinas en la enseñanza real de la historia, hay que considerar que estas últimas, pese a lo que tengan de supervivencias y de arcaísmos, contienen en sí mismas una racionalidad o lógica institucional y adoptan la forma de estructuras de larga duración enmarcadoras de la acción docente de los profesores de historia. La vieja enseñanza de la historia ha perdido legitimidad, pero no vigencia $(2011$, p. 82)

Esto tendría que relacionarse con las prácticas que tienen lugar dentro del aula. En relación al profesor, este sigue teniendo un papel protagonista como transmisor de los contenidos que tiene que adquirir el alumnado. Así, es la explicación del profesor lo que predomina frente a otras posibles estrategias como bien pudiera ser la heurística, el procesamiento de la información por los estudiantes o la socialización con problemas de actualidad (Moreno Vera, Vera Muñoz, y Martínez Valcárcel, 2015). Esto casaría con lo que podríamos denominar como un modelo didáctico transmisivo (García Pérez, 2000), según el cual los contenidos se conciben desde una óptica enciclopedista y estos deben ser adquiridos por el alumnado a través de las explicaciones del profesor. Así, pues, el conocimiento se presenta como una "verdad" acabada y los estudiantes deberán demostrar su aprendizaje por medio del examen, por lo que prima la evaluación final.

La enseñanza de la historia se presta en gran medida a este modelo, pues como señalan algunos estudios, los docentes cuentan entre sus principales aliados con el libro de texto, sus actividades y el examen. En el caso de la historia, los manuales escolares españoles parecen responder a una exposición lineal del relato histórico desde un punto de vista "rankeano", esto es, teniendo un peso 
primordial los acontecimientos y contenidos de índole política, aunque complementados con otros contenidos centrados en el arte y la cultura, la sociedad o la economía. Este relato, a su vez, responde a una óptica centrada en la construcción nacional, que se articula en base a esos grandes acontecimientos de la historia de España. En cualquier caso, los contenidos se presentan de una forma descriptiva y factual, como partes independientes y estancas de una historia mayor o general (Gómez Carrasco y Chapman, 2017, p. 353). De esta manera, la explicación del profesor estaría ligada a los contenidos expresados en los libros de texto.

Si seguimos en esta misma línea, Sáiz (2011) realizó un estudio de las actividades de los libros de texto de historia. Según este autor, la mayoría de estas actividades exigen un nivel cognitivo bajo, ya que se trata de buscar y reproducir las respuestas que aparecen en el propio manual. Por su parte, Gómez Carrasco (2014) indica que los libros de texto siguen mostrando una visión lineal y no actualizada del pasado, y las actividades se basan en la repetición de hechos, fechas o datos concretos. En otras palabras, este tipo de actividades, si bien fomentan el desarrollo de la competencia memorística, no propician una "correcta alfabetización histórica del alumnado" (2014, p. 155).

En este panorama se entiende el uso extendido que tiene la práctica examinatoria en la clase de historia. En efecto, al primar la memorización de unos contenidos conceptuales, se opta por el examen como principal (a veces casi único) instrumento de evaluación. Tal es así que el profesorado acaba por darle más importancia a la cantidad de información que a su calidad (Gómez Carrasco y Miralles Martínez, 2015). El examen constituye una prueba objetiva del conocimiento escolar (PérezGuerrero, 2016), algo básico dado las presiones que recaen sobre el profesorado en relación a la necesidad de certificación de los estudiantes (Miralles Martínez, Molina Puche, y Monteagudo Fernández, 2017) o a la evaluación desde las instituciones e informes internacionales. Pero, además, en el desarrollo de la clase prevalece otro aspecto primordial como es el control de la misma, de modo que el examen se presta como un instrumento de control para el profesorado. Esto, junto con lo esbozado más arriba (la preeminencia de la explicación del profesor, las actividades, que en cierto modo constituyen un ensayo antes de la prueba final, y el examen) favorecen el gobierno de la clase, tal como indica en sus estudios Merchán (2001, 2005, 2011).

En definitiva, son numerosos los estudios que han tratado de analizar el funcionamiento de las clases de historia. No obstante, es evidente que la enseñanza de la historia en la etapa secundaria ofrece una mayor potencialidad formativa para el alumnado y las investigaciones han demostrado que existen nuevos caminos por recorrer. A este respecto, a pesar de la permanencia de la tradición, la innovación educativa en la didáctica de la historia está cada vez más presente en las aulas.

\section{LA NECESIDAD DE DESARROLLAR EL PENSAMIENTO HISTÓRICO COMO BASE DE LA ENSEÑANZA}

Las dinámicas de la clase de historia esbozadas más arriba no propician el desarrollo del pensamiento histórico y crítico. De esta manera, en general, lo que se fomenta es el aprendizaje, por medio de la memorización, de contenidos de tipo conceptual. A este respecto, numerosas investigaciones manifiestan la existencia de distintos tipos de competencias y contenidos históricos a enseñar. Así, podemos hablar de contenidos de primer orden, esto es, son los que hacen referencia a los aspectos factuales (hechos o acontecimientos históricos) y cronológicos. Como hemos visto, estos son los que tienen una mayor presencia en las clases de historia. Ahora bien, también conviene considerar los denominados contenidos de segundo orden o de carácter estratégicos, que son aquellos relacionados con las competencias del historiador y el desarrollo del pensamiento histórico. Además, estos contenidos permiten la organización y comprensión de los primeros (Gómez Carrasco y Miralles Martínez, 2015, p. 54). Podemos verlo mejor en la siguiente tabla (Tabla 1): 
TABLA 1. Contenidos y competencias históricas

\begin{tabular}{|c|l|}
\hline \multicolumn{2}{|c|}{ Historia sustantiva o de primer orden } \\
\hline Cronología & $\begin{array}{l}\text { Se exige conocimiento sobre las fechas en las que ocurrieron } \\
\text { los procesos históricos o saber ubicarlos de forma correcta. }\end{array}$ \\
\hline Historia estratégica o de segundo orden \\
\hline \multicolumn{1}{|c|}{$\begin{array}{l}\text { Se exige conocimiento de un concepto o un hecho concreto } \\
\text { del pasado }\end{array}$} \\
\hline Relevancia histórica & $\begin{array}{l}\text { Explicar la relevancia histórica de un evento o persona en } \\
\text { particular utilizando criterios apropiados. }\end{array}$ \\
\hline Fuentes/evidencias & $\begin{array}{l}\text { Entender como la historia es una interpretación basada en } \\
\text { inferencias a partir de fuentes primarias. }\end{array}$ \\
\hline Cambio y continuidad & $\begin{array}{l}\text { Comprender el cambio en el pasado como un proceso, con } \\
\text { diferentes ritmos y patrones. Identificar los patrones } \\
\text { complejos del proceso y decadencia en los diferentes pueblos } \\
\text { o sociedades. }\end{array}$ \\
\hline Pausas y consecuencias & $\begin{array}{l}\text { Reconocer múltiples causas y consecuencias a corto y largo } \\
\text { plazo. Ver la consecuencia de un hecho o persona concreta } \\
\text { en las actividades humanas y las estructuras y condiciones } \\
\text { actuales }\end{array}$ \\
\hline Dimensión ética & $\begin{array}{l}\text { Reconocer las diferencias entre las actuales creencias, } \\
\text { valores y motivaciones (visión del mundo) y la de los } \\
\text { pueblos y sociedades anteriores. Explicar las perspectivas de } \\
\text { las personas en el pasado en su contexto histórico. }\end{array}$ \\
\hline $\begin{array}{l}\text { Realiza juicios éticos razonados sobre acciones de la gente } \\
\text { en el pasado, reconociendo el contexto histórico en el que } \\
\text { operaban. Evaluar las implicaciones en el presente de los } \\
\text { sacrificios e injusticias del pasado. }\end{array}$ \\
\hline
\end{tabular}

Fuente: Gómez Carrasco y Miralles, 2017

Estas son, pues, las competencias históricas que se pretenden trabajar. Se trata de romper con esa concepción estanca y meramente descriptiva de la historia para poder comprender el proceso de construcción del conocimiento histórico. Así pues, conviene diferenciar entre los estudiantes que historia y pasado no son lo mismo. El pasado constituye el objeto de estudio de la historia, pero constituye una realidad difícil de reconstruir, pues a diferencias de otras ciencias experimentales, no se puede constatar empíricamente todo lo que ocurrió. Así pues, la historia permite realizar una reconstrucción de ese pasado de la humanidad mediante una metodología propia de la disciplina, basada en el estudio de las fuentes y en su interpretación (Gómez Carrasco, Miralles Martínez, y Molina Puche, 2015). Por esta razón, hay que destacar la importancia de que los alumnos y alumnas trabajen con fuentes históricas y comprendan la función del método científico de la historia.

Esta función incide en que los alumnos aprendan los métodos y las técnicas que utilizan los historiadores para conocer el pasado de forma rigurosa. Esto supone que los jóvenes deben aprender a formular hipótesis sobre los hechos que deben analizar, buscar explicaciones a través de distintas fuentes de información y construir generalizaciones y modelos que den explicación al problema planteado y otros de similar estructura (Prieto Prieto, Gómez Carrasco y Miralles Martínez, 2013, p. 3)

De esta forma, se trabajan competencias asociadas al pensamiento histórico, tales como la aplicación del método del historiador. En realidad, se trata de fomentar la investigación del alumnado sobre un problema de tipo histórico que puede plantear el profesorado. Esto puede conectarse con los nuevos enfoques constructivistas en los que el alumnado va adquiriendo un mayor protagonismo o con el modelo didáctico de investigación en la escuela (García Pérez, 2000; García Pérez y Porlán, 
2000). Para ello, se pueden utilizar estrategias tales como el Aprendizaje Basado en Problemas, estudios de caso, proyectos... que proponen la solución de un problema por medio de la formulación de hipótesis o estrategias de indagación. Pero, del mismo modo, se propone que el resto de actividades que realiza el alumnado respondan precisamente a las diversas competencias históricas, como las que hemos visto, no solo a la factual o cronológica.

En este mismo orden de cosas, se puede destacar la potencialidad de la narración como instrumento para la representación del pasado y el desarrollo el pensamiento histórico. De hecho, el mismo Elliott destacaba como el ensayo constituía una de las principales herramientas para favorecer la reflexión histórica (2012). La narración permite reflexionar sobre el pasado (no solo describir el pasado, sino explicarlo) y comprender que el relato histórico es una construcción a partir de las evidencias estudiadas. Igualmente, permite seguir la progresión del aprendizaje del alumnado y su capacidad para organizar y comprender lo estudiado. Así, pues, puede considerarse un indicador de la madurez del pensamiento (Gómez Carrasco y Miralles Martínez, 2015, pp. 65-66). De esta forma, la evaluación por competencias (centradas en los distintitos tipos de contenido histórico) puede favorecer un seguimiento, de manera continua, de los progresos del alumnado.

Finalmente, conviene añadir la importancia de la educación para la ciudadanía (García Pérez y De Alba, 2008; Navarro-Medina y De-Alba-Fernández, 2011). La formación histórica ofrece una estrecha relación con el desarrollo de una participación ciudadana crítica, esto es, la enseñanza de la historia y de su disciplina puede dotar de una serie de herramientas a los estudiantes para comprender el mundo que les rodea e identificarse con la participación en la sociedad. Para ello, evidentemente, es necesario que desde la materia se trabaje el desarrollo de capacidades cognitivas complejas de análisis e interpretación, así como de las competencias sociales y cívicas (Gómez Carrasco et al., 2015). En este sentido, el pensamiento histórico favorece la formación cívica y constituye uno de los principales objetivos de la enseñanza de la historia (Pagès, 2007; Santiesteban y Pagès, 2010).

Teniendo en cuenta todo lo anterior, se puede colegir la importancia que tiene la historiografía (método y tarea del historiador, objetivo del estudio del pasado, utilidad de la disciplina...) en la enseñanza de la historia, puesto que el alumnado debe manejar estas nociones para poder desarrollar el pensamiento histórico. Es por ello que creemos necesario estudiar cómo evolucionan las concepciones del alumnado sobre el conocimiento historiográfico con una metodología que tenga en cuenta los aspectos señalados, ya que esto favorecerá la adquisición de las distintas competencias históricas.

\section{LAS CONCEPCIONES DEL ALUMNADO SOBRE EL CONOCIMIENTO HISTÓRICO}

Llegados a este punto, hemos de destacar la importancia de conocer las visiones del alumnado respecto a la materia de enseñanza. De hecho, las investigaciones que convergen en este aspecto se han mostrado de gran utilidad. En concreto, Concha Fuentes destaca el interés de conocer las concepciones sobre la historia para enfrentar "la dificultad de trabajar los conceptos básicos de historia con grupos de adolescentes que emplean esquemas de razonamiento muy complejos y en los que pesan de forma sustancial los tópicos o ideas erróneas sobre el pasado" (2002, p. 55). En efecto, en la actualidad las visiones de los estudiantes sobre la historia están influidas por elementos tan presentes en sus vidas como el cine, la literatura, los videojuegos, redes sociales...que ofrecen una imagen que no siempre concuerda con la realidad histórica aceptada (López Facal, 1999). En este sentido, difícilmente la escuela puede competir con los medios de comunicación. Ahora bien, no se trata de sustituir de raíz estas visiones. Desde nuestra óptica, es aquí donde cobra valor el estudio de la historiografía como herramienta para poner en conflicto las ideas previas del alumnado y transitar hacia una visión o ideas más complejas en consonancia con el pensamiento histórico. Una buena manera de aproximarse a esta realidad es por medio de la investigación educativa.

A este respecto, Valls y López Facal (2011) expusieron que, desde el punto de vista de los estudiantes, la historia se entendía como el estudio de una serie de hechos y la memorización de los 
mismos. Además, la utilidad de la historia se reducía a la adquisición de cultura, vía para la distinción social. Esto último recuerda a la noción de "capital cultural" que ya planteó Bourdieu.

No obstante, dado nuestro foco de interés, hemos de mencionar la importancia de los trabajos de Concha Fuentes $(2002,2004)$, donde estudió las concepciones del alumnado sobre el conocimiento histórico y la historia escolar. En nuestro caso, nos hemos centrado en sus aportaciones sobre las concepciones acerca del conocimiento histórico y extraemos algunas de sus ideas clave que pudieran servir para los objetivos de nuestra investigación:

- En general, el alumnado asocia la historia con el estudio del pasado referido a los grandes acontecimientos y las hazañas de personajes relevantes.

- Los estudiantes consideran que el objeto del estudio de la historia es el pasado de la humanidad en su conjunto.

- El alumnado presenta dificultades notorias para explicar el trabajo del historiador y suele confundirlo con la narración descriptiva del pasado, sin considerar el elemento interpretativo. No obstante, en aquellos alumnos que trabajan con fuentes, señalan la importancia de las mismas para la reconstrucción del pasado.

- Para los estudiantes, el interés y la utilidad de la historia reside en la adquisición de una "mayor cultura".

\section{Metodología}

Para la realización de esta investigación se ha elaborado una metodología en la que se trabajen los contenidos y competencias históricas para favorecer el pensamiento crítico y el desarrollo de estructuras cognitivas más complejas de las que se suelen trabajar en las dinámicas de clase más tradicionales. Con ello se busca fomentar la aplicación del pensamiento histórico y critico como forma de comprender la sociedad y fomentar la participación ciudadana. A partir de aquí, el objetivo principal es conocer cómo evolucionan las ideas historiográficas del alumnado siguiendo esta metodología. A este respecto, Fuentes (2004) ya señaló que la visión del alumnado sobre la historia estaba influenciada por el tipo de didáctica aplicada en el aula. De esta manera, podemos formular la siguiente pregunta de investigación: ¿Cómo evolucionan las ideas historiográficas del alumnado de $1^{\circ} \mathrm{ESO}$ con una metodología basada en el trabajo de las competencias asociadas al pensamiento histórico?

En este trabajo se ha optado por un enfoque de tipo cualitativo, para poder comprender como se produce el cambio en las concepciones del alumnado sobre el conocimiento histórico. Para ello, se llevó a cabo una estrategia plurimetodológica que constó de dos niveles de análisis: un estudio descriptivo y un análisis de casos múltiples.

Respecto a los participantes y al contexto de la investigación, se ha trabajado con una muestra de un grupo con 23 alumnos y alumnas de $1^{\circ} \mathrm{ESO}$, entre los cuales se seleccionaron tres casos que resultaron significativos para el objeto de la investigación. Esta investigación hay que contextualizarla en un IES de la provincia de Almería. Se trata de un grupo de un nivel cultural bajomedio que trabajó los contenidos históricos correspondientes a su nivel escolar. Para favorecer el desarrollo del pensamiento histórico (Gómez Carrasco, 2014; Prieto Prieto et al., 2013), se ha optado por diseñar una metodología de trabajo en la que en cada unidad didáctica se ofrece un caso a los estudiantes que tienen que resolver. Se trata de un problema formulado a modo de pregunta que el alumnado tiene que responder por medio del análisis de distintas fuentes históricas. A partir del trabajo con las fuentes, el alumnado debe resolver el problema histórico planteado y realizar una narración histórica en la que reconstruya el caso por medio de la propia interpretación de las evidencias estudiadas. Así, por ejemplo, para la primera unidad del bloque se le plantea un interrogante sobre el hallazgo de un yacimiento prehistórico con varias fuentes que indican un modo de vida. Los estudiantes analizarán las distintas fuentes (cerámica neolítica, hoces de piedra, restos de ganado...) para ubicar cronológicamente el yacimiento y reconstruir, por medio de la narración, 
como hubo de ser la vida en ese yacimiento en base a las evidencias y los conocimientos que tienen sobre la etapa. La unidad se completa con la realización de un dossier de actividades relacionadas con las distintas competencias históricas (relevancia histórica, causas y consecuencias, cambio y continuidad...) y los estándares de aprendizaje correspondientes. Estas preguntas asociadas a las competencias históricas son las que se trabajarían en la prueba escrita.

La evaluación tanto del dossier, la resolución del caso y la prueba escrita se realiza con unas rúbricas elaboradas a tal fin, con una serie de indicadores de logro. Lo que se evalúa, por tanto, es la aplicación de las competencias sobre los contenidos históricos trabajados, lo que permite hacer un seguimiento continuo del progreso del alumnado a lo largo de las unidades didácticas. Paralelamente, los estudiantes participan en un proyecto interdisciplinar consistente en la elaboración de un museo. En historia, por medio del trabajo cooperativo, realizaron una serie de maquetas basadas en producciones artísticas, monumentos o reconstrucciones históricas de las etapas trabajadas, teniendo en cuenta sus características, funciones, etc. Asimismo, elaboraron una guía en la que se explican todos estos aspectos. Además, los grupos realizaron pequeñas obras de teatro, trabajando, de esta forma, la empatía histórica.

TABLA 2. Niveles de progresión

\begin{tabular}{|c|c|c|c|c|}
\hline & Nivel 0 & Nivel 1 & Nivel 2 & Nivel 3 \\
\hline $\begin{array}{c}\text { Visión de la } \\
\text { historia }\end{array}$ & $\begin{array}{l}\text { Es incapaz de } \\
\text { expresar su su } \\
\text { visión de la } \\
\text { historia ni qué o } \\
\text { a quién estudia }\end{array}$ & $\begin{array}{l}\text { Identifica la historia } \\
\text { con lo ocurrido en el } \\
\text { pasado o los recuerdos } \\
\text { y centrada en los } \\
\text { personajes } \\
\text { relevantes/antepasados }\end{array}$ & $\begin{array}{l}\text { Considera la historia } \\
\text { como el estudio de los } \\
\text { acontecimientos y } \\
\text { hechos del pasado de } \\
\text { los antepasados, } \\
\text { centrándose en los } \\
\text { personajes más } \\
\text { relevantes }\end{array}$ & $\begin{array}{lr}\text { Considera } & \text { la } \\
\text { historia como el } \\
\text { estudio } & \text { e } \\
\text { interpretación del } \\
\text { pasado de la } \\
\text { humanidad } \\
\text { (sociedad, política, } \\
\text { economía y cultura) }\end{array}$ \\
\hline $\begin{array}{c}\text { Objetivo de la } \\
\text { historia }\end{array}$ & $\begin{array}{lr}\text { Es incapaz } & \text { de } \\
\text { definir } & \text { los } \\
\text { objetivos de la } \\
\text { historia }\end{array}$ & $\begin{array}{l}\text { Considera que el } \\
\text { objetivo de la historia } \\
\text { es recordar las grandes } \\
\text { hazañas del pasado y } \\
\text { recordar los aspectos } \\
\text { más interesantes del } \\
\text { pasado a } \\
\begin{array}{l}\text { transmitirlo a la } \\
\text { sociedad }\end{array}\end{array}$ & $\begin{array}{l}\text { Considera que el } \\
\text { estudio de la historia } \\
\text { consiste en estudiar la } \\
\text { historia general de la } \\
\text { humanidad para no } \\
\text { repetir los errores del } \\
\text { pasado }\end{array}$ & $\begin{array}{lrr}\text { Considera que } & \text { el } \\
\text { estudio de } & \text { la } \\
\text { historia } & \text { es } \\
\text { comprender } & \text { los } \\
\text { cambios en las } \\
\text { sociedades a lo } \\
\text { largo del tiempo }\end{array}$ \\
\hline $\begin{array}{c}\text { Tarea del } \\
\text { historiador }\end{array}$ & $\begin{array}{l}\text { Desconoce } \\
\text { quien estudia la } \\
\text { historia y como } \\
\text { se estudia el } \\
\text { pasado }\end{array}$ & $\begin{array}{l}\text { Lo confunde con el } \\
\text { arqueólogo, científico } \\
\text { o profesor y cree que el } \\
\text { conocimiento histórico } \\
\text { es una verdad acabada } \\
\text { que aparece en los } \\
\text { libros o se transmite de } \\
\text { generación en } \\
\text { generación }\end{array}$ & $\begin{array}{lr}\text { Identifica } & \text { al } \\
\text { historiador, aunque lo } \\
\text { confunde con el } \\
\text { arqueólogo } \\
\text { científico, y considera } \\
\text { que su trabajo consiste } \\
\text { en recapitular } \\
\text { información a través de } \\
\text { libros, testigos (visión } \\
\text { parcial de las fuentes) }\end{array}$ & $\begin{array}{l}\text { Identifica la tarea } \\
\text { del historiador con } \\
\text { el análisis de las } \\
\text { fuentes y su } \\
\text { interpretación para } \\
\text { elaborar un relato } \\
\text { histórico del pasado } \\
\text { (método r del } \\
\text { historiador) }\end{array}$ \\
\hline $\begin{array}{c}\text { Utilidad de la } \\
\text { historia }\end{array}$ & $\begin{array}{l}\text { Es incapaz de } \\
\text { expresar utilidad } \\
\text { alguna }\end{array}$ & $\begin{array}{l}\text { Considera que la } \\
\text { utilidad de la historia es } \\
\text { la adquisición de } \\
\text { información cultural } \\
\text { para obtener prestigio } \\
\text { social e informar a los } \\
\text { demás }\end{array}$ & $\begin{array}{l}\text { Considera que la } \\
\text { utilidad de la historia } \\
\text { radica en recordar los } \\
\text { aspectos más signi- } \\
\text { ficativos del pasado y } \\
\text { mejorar la vida actual } \\
\text { en base a ello }\end{array}$ & $\begin{array}{l}\text { Considera que la } \\
\text { utilidad de la } \\
\text { historia radica en } \\
\text { permitir compren- } \\
\text { der mejor la } \\
\text { sociedad actual y su } \\
\text { participación en ella }\end{array}$ \\
\hline
\end{tabular}

Fuente: Elaboración propia 
En relación a los instrumentos, en primer lugar, se realizó un estudio descriptivo por medio de un cuestionario inicial basado en el utilizado por Concha Fuentes $(2002,2004)$ en sus estudios sobre las concepciones del alumnado, si bien, en nuestro caso, nos hemos centrado en las preguntas sobre el conocimiento histórico y lo hemos adaptado al problema de nuestra investigación. Las preguntas respondían a cuatro primeras categorías básicas, a saber: visión sobre la historia; objeto de estudio de la historia; tarea del historiador; y utilidad de la historia. El cuestionario constaba de preguntas abiertas y cerradas, lo que permitía la adquisición de unidades de información. Las respuestas se agruparon en función a su semejanza, estableciendo categorías emergentes dependiendo del tipo de respuesta. Un nuevo cuestionario se realizó al final del proceso de enseñanza aprendizaje, lo que permitió realizar una comparación de las respuestas en dos momentos diferentes (ideas previas/ideas finales). Para facilitar el seguimiento del alumnado, se establece unas hipótesis o niveles de progresión (Tabla 2), que se presentan como un instrumento de gran utilidad para la intervención e investigación educativa (Martínez Rivera y Martínez Rivera, 2012). En un segundo nivel de análisis, y atendiendo a las respuestas de los cuestionarios, se ha optado por un análisis de casos múltiple, seleccionando a aquellos alumnos cuyas respuestas pudieran ser más significativas. De esta manera, se realizaron entrevistas de carácter semiestructurado a tres estudiantes para profundizar, contrastar y complementar la información obtenida en los cuestionarios.

Pata analizar los datos, se agruparon las respuestas obtenidas en los cuestionarios y se relacionaron con las categorías establecidas para el análisis, identificándolo con uno de los niveles de progresión, lo que permitió una cuantificación y representación gráfica de las respuestas. Para los datos obtenidos de las entrevistas, se utilizó un análisis de contenidos, cruzando las unidades de información más significativas con los resultados obtenidos en los cuestionarios. Con ello se pretende obtener una visión panorámica de la evolución de las concepciones historiográficas del alumnado y comprender que aspectos han podido incidir en los cambios experimentados.

\section{ANÁLISIS}

Los cuestionarios iniciales nos sirvieron para explorar las ideas previas que presentaba el alumnado sobre el conocimiento histórico. Las respuestas se agruparon según el nivel de semejanza teniendo en cuenta las categorías trabajadas. De esta manera, en sus respuestas respecto a la visión de la historia, la mayoría de los alumnos la entendían como el estudio de los hechos del pasado (39,1\%), seguido por la identificación de la historia con el pasado $(21,7 \%)$ y, en tercer lugar, lo entendían como la memoria o recuerdo de lo que ocurrió (13\%). Además, para la mayoría, la historia estudia los grandes acontecimientos del pasado (47,8\%), seguido en segundo y tercer lugar por las respuestas que se refieren al estudio del pasado en general y al estudio de los antepasados (13\%). Otras respuestas se referían al estudio del tiempo o de los objetos (cultura material). El 17,3\% no sabía qué estudia la historia.

El cuadro se completa cuando se le pregunta a quién estudia la historia. Aquí destacan las respuestas referidas a los personajes relevantes $(69,5 \%)$, seguidas de otras respuestas minoritarias (monos, pueblos, antepasados, humanidad). De esta manera, parece desprenderse la idea de que la mayoría de los estudiantes entienden la historia como el estudio de los hechos del pasado, -aunque un porcentaje importante confunde pasado e historia-, centrado en el estudio de los grandes acontecimientos protagonizados por personajes importantes.

Respecto al objetivo de la historia, destacan las respuestas que consideran que el objetivo es informar y enseñar el pasado (30,4\%), seguidas de las que consideran que el objetivo es entender el pasado $(21,7 \%)$ y conocer la evolución de los antepasados $(17,3 \%)$. El 13\% considera que el objetivo es aprender del pasado.

En lo referente a la tarea del historiador, el 26\% señala que el historiador es quien se encarga de estudiar el pasado histórico, seguido del científico $(21,7 \%)$, el arqueólogo $(17,3 \%)$ y el profesor 
(13\%). El 21,7\% admite no saberlo. Así, resulta llamativo el alto grado de imprecisión mostrado por los estudiantes. Esto se muestra muy visible cuando preguntamos cómo se estudia el pasado. El 26\% desconoce cómo se hace, mismo porcentaje para aquellos que señalan que el pasado se estudia mediante el análisis de fuentes o "pistas" y elaborando hipótesis. El resto piensa que se realiza por medio de la búsqueda de información (libros, internet), por medio de testigos y la transmisión oral o con algún tipo de investigación que desconocen.

Finalmente, en lo que se refiere a la utilidad de la historia, la mayoría piensa que es útil, pero no puede precisar para qué $(30,4 \%)$. Le siguen las respuestas que consideran que la historia es útil para obtener cultura $(21,7 \%)$ y para aprender del pasado (17,3\%). Otro $21,7 \%$ no sabe si la historia tiene utilidad.

Pues bien, al final del trimestre, después de trabajar con la metodología descrita, se volvió a pasar un cuestionario final al alumnado. En él podemos apreciar algunos cambios significativos.

En lo relativo a la visión de la historia, la mayoría del alumnado sigue considerando que se trata del estudio de los hechos del pasado (26\%), mientras que un $21 \%$ suele confundir la historia con el pasado y un $13 \%$ la considera como el estudio de los hechos y estructuras del pasado. No obstante, si se les pregunta qué estudia la historia, el 56\% afirma que estudia aspectos como la sociedad, economía, política...El resto de respuestas son minoritarias, salvo la de aquellos que piensan que la historia estudia el cambio en las sociedades (13\%). A esto se le suma que el $42 \%$ piensa que la historia se centra en estudiar a la humanidad, aunque aún muchos piensan que la historia investiga sobre los personajes más importantes $(30 \%)$. De esta manera, vemos como hay cierto cambio al considerar dentro del estudio del pasado las estructuras y al ser humano, frente a aquella visión más restringida al hecho y personajes relevantes como reyes, nobles...De hecho, en las entrevistas los alumnos encuestados explican cómo han cambiado sus ideas:

Sí, muchísimo, pues yo por ejemplo antes me creía que la historia era todo en general, como los dinosaurios, como las plantas o cualquier cosa y nosotros los seres humanos. Pero ahora sí ha cambiado porque sé que la prehistoria era antes de que apareciera la escritura... que se trata solo... que el protagonista es el ser humano (EntrAlum21)

Sí, porque antes no sabía nada de historia y ahora me he enterado de todo y es como ¡guay!, lo sé (...) Pues no sé, decía que era los dinosaurios (...) Pues me has ido explicando cosas, yo las he ido entendiendo y ya he ido diciendo, ¡ah! Esto tiene más sentido. (EntrAlum20)

Para los objetivos de la historia, las respuestas nos indican que el alumnado considera de vital importancia conocer los cambios acaecidos en las sociedades (34\%), así como entender el pasado para cambiar el presente $(21,7 \%)$ y comprender el pasado $(17 \%)$. De este modo, parece superarse aquella otra visión que considera informar cómo el objetivo de la historia, dando mucha más importancia a la compresión de los cambios en la sociedad: "antes pensaba que la historia, no sé, no servía. Ahora creo que para ver la evolución, sobre todo" (EntrAlum20)

La mayor evolución se ha percibido en relación al conocimiento sobre la tarea del historiador. La gran mayoría del alumnado reconoce al historiador o arqueólogo como aquel que investiga el pasado (87\%) por medio del análisis e interpretación de fuentes históricas de diverso tipo (87\%). Tan solo el $13 \%$ tiene dudas sobre quien estudia la historia y como lo hace. Esta idea se confirma en las entrevistas.

Yo pensaba que... no sé. Que encontraban cosas, las iban juntando y tenían una pista o algo. Ahora sé que es través de las fuentes, ahora sé cómo se llaman todas esas cosas (EntrAlum20)

Pues como era exactamente lo de las fuentes históricas (...) Ahora sé que no se lo inventan, digamos, porque a ver, no me creo que se lo hayan inventado en un principio, pero puede haber algo que sí. Pero ahora sé mejor que intentan ver lo más real posible, que no sea tan mitológico (EntrAlum21) 
En las entrevistas, se le preguntó al alumnado qué creían que había influido para cambiar sus ideas sobre el conocimiento de la tarea del historiador. En ellas, destacan el trabajo con las fuentes en los casos prácticos planteados.

Los casos nos han hecho ser como historiadores o arqueólogos, hemos ido como juntando piezas, y hemos formado un caso, ¿sabes? Eso me ha ayudado a que mi texto tenga coherencia (EntrAlum20)

Los casos. El caso era una ficha donde teníamos que buscar en concreto una cosa y las fuentes podían ser escritas, materiales... y como que cada fuente era una pequeña pieza del puzle y nosotros teníamos que decir que nos transmitía esa fuente. Y con todas esas fuentes hacíamos como una redacción diciendo que podíamos saber que hacían antes (EntrAlum21)

Finalmente, en lo que se refiere a la utilidad de la historia, el alumnado considera que su utilidad radica en comprender cómo éramos antes y de dónde venimos (26\%) y para aprender del pasado $(26 \%)$, aunque es reseñable que un $21 \%$ sigue teniendo dudas respecto a la utilidad de la historia. En este sentido, se aprecia una evolución en las concepciones, en tanto que casi el 50\% entiende que existe una utilidad en la historia, si bien un porcentaje significativo no lo ve claro. Destacamos algunas respuestas significativas del cambio en esta categoría:

Sí, porque yo pensaba que la historia no servía de nada, que era como para meternos un poquito la jeta. Pero no, así podemos aprender de dónde venimos, quienes somos y de donde procedemos (EntrAlum17)

Antes pensaba que la historia, no sé, no servía. Ahora creo que, para ver la evolución, sobre todo (EntrAlum20)

Sinceramente sí, pensaba que aprender era personal, que podía aprender yo por mí misma tipo sí yo sé, pero ya está, solo por saber. Pero creo que sí es útil que mucha gente sepa, como has dicho, que tenga el nivel básico de la historia, no estaría mal. (EntrAlum21)

Si comparamos la evolución de las respuestas en los cuestionarios iniciales y finales (véase gráfico 1), apreciamos una evolución positiva del alumnado.

GRÁFICO 1. Comparación de los niveles inferidos en los cuestionarios inicial y final
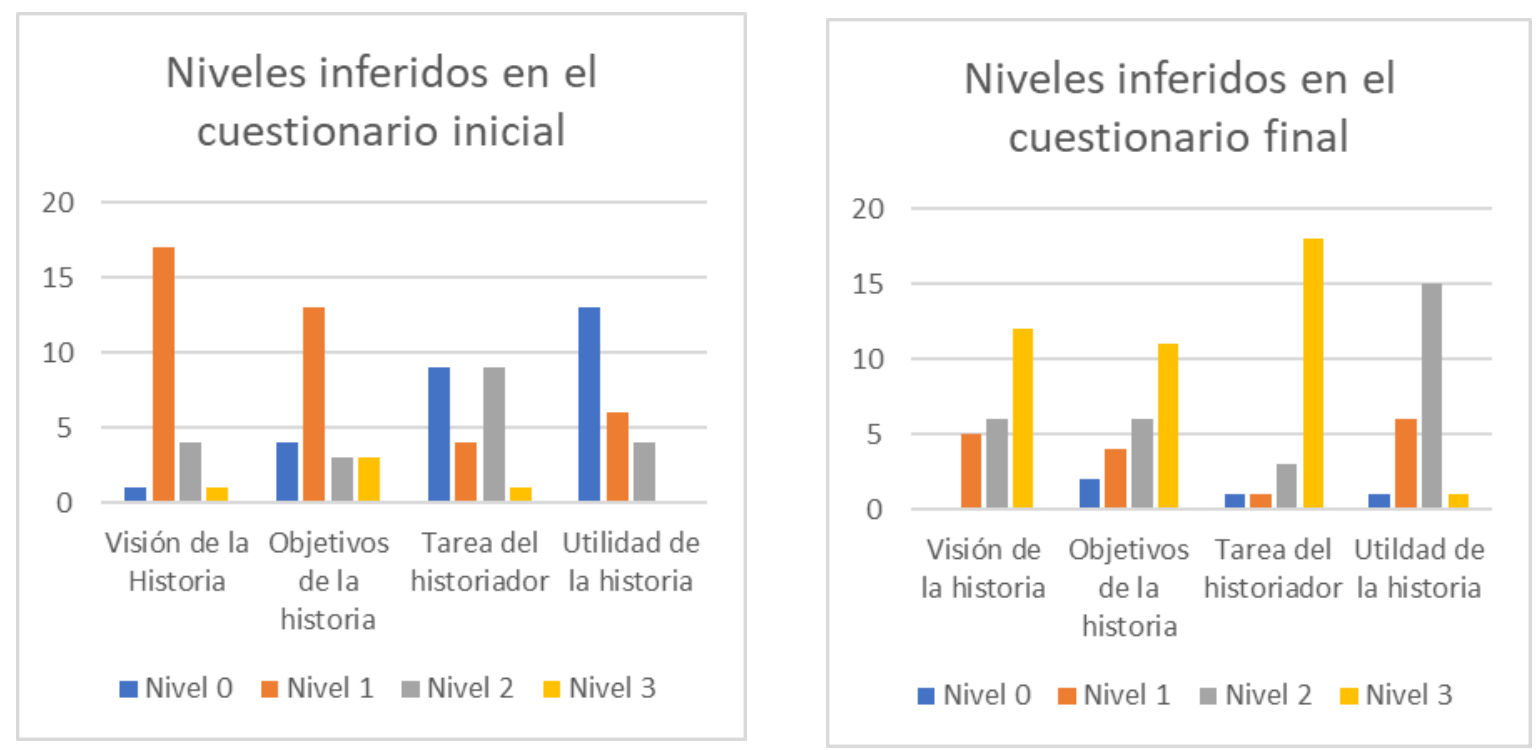
Los niveles 2 y 3 se muestran más bajos en el primer gráfico, mientras que en el segundo aumenta su presencia notablemente. Esto es especialmente evidente en los referido a la tarea del historiador, donde un porcentaje alto de los alumnos alcanza el nivel 3. Esta evolución, aunque menor, también es destacada en la visión de la historia y los objetivos de la historia. Ahora bien, donde más dificultades se ha encontrado es en lo referido a la utilidad de la historia. Aquí si ha habido evolución, pero solo un pequeño porcentaje del alumnado ha alcanzado el nivel 3. Esto puede explicarse por el punto de partida, puesto que una buena parte de los estudiantes desconocían cualquier utilidad de la historia o lo veían simplemente como una adquisición cultural, por lo que los niveles de partida eran más bajos en este apartado que en cualquier otro.

Si nos preguntamos qué aspectos han podido incidir en los cambios experimentados entre el alumnado, podemos encontrar algunas respuestas en las entrevistas. En este sentido, habría que destacar las actividades desarrolladas en clase, entre las que destacan algunas como las referidas a la empatía histórica:

Pues, por ejemplo, cuando estábamos haciendo las actividades y sale alguna de ponerse en la piel del otro y ahí como que recapacitas para ver lo que hacían y todo eso. Entonces ya conoces más su vida (EntrAlum17)

Sí, meterme como en el personaje, pues pensaba que no era así como pensaban ellos (...) Pues no sabía cómo pensaban antes y ahora pensamos de otra manera (EntrAlum20)

Los casos también han contribuido al cambio de las ideas del alumnado según estos, lo que puede explicar la importante evolución respecto a las concepciones sobre la tarea del historiador.

Que tú nos ibas dejando como pistas y nosotros íbamos adivinando eso, y como que nos dejabas que nosotros explicásemos lo que iba sucediendo (EntrAlum20)

Otros aspectos que han valorado positivamente en su aprendizaje han sido la posibilidad de emitir juicios de opinión sobre los aspectos históricos tratados, ubicándolos en el tiempo, e, igualmente, la metodología basada en preguntas al grupo para que, a partir de sus respuestas, se fuese construyendo el conocimiento (método mayéutico).

Las actividades. Los textos y poner la opinión personal, ponerse en la piel de las demás y los ejes cronológicos (EntrAlum17)

Pues de la manera que lo has explicado, cómo hemos colaborado, tú ibas preguntando cosas mínimas y nosotros íbamos alumno por alumno como juntando un puzle, cada uno era una pieza y uno decía una cosa, el otro otra y quiera o no pues te quedas con las cosas (EntrAlum21)

Incluso se podría destacar la opinión de uno de los alumnos entrevistados, que opinaba que este tipo de metodología favorecía un aprendizaje significativo.

Me ha gustado un montón las clases, como podemos aprenderlo. Saber que yo sé perfectamente que el año que viene voy a saber todo. Ósea, si me preguntan yo voy a empezar por el Paleolítico y voy a saber todo. Y entonces, eso anteriormente pues yo lo había aprendido y era como que yo, por ejemplo, estudié, lo di el año pasado, pero no me acordaba de nada, porque era apuntes y apuntes... y apuntes. Pero ahora sé que me voy a quedar con, por lo menos, la base, toda mi vida. (EntrAlum21) 


\section{Conclusiones}

En definitiva, para la realización de esta investigación se ha intentado programar una metodología práctica y real en la que se trabajen aquellos contenidos y competencias que favorezcan el desarrollo del pensamiento histórico, siendo esta la referencia principal (Gómez Carrasco y Miralles Martínez, 2017). A partir de ahí, se trataba de comprobar si esta metodología, que busca un papel activo del alumnado, podía favorecer el cambio y la evolución de las concepciones historiográficas, ya que estas se antojan fundamentales para la alfabetización histórica de los estudiantes.

Los cuestionarios iniciales confirman algunas aportaciones de investigadores, tales como que el alumnado entiende la historia como el estudio de los hechos del pasado, basado en los grandes acontecimientos y personajes más relevantes, no entiende de manera precisa como se construye el conocimiento histórico o considera la historia como un medio para conseguir prestigio cultural (Fuentes, 2004).

No obstante, de acuerdo con las respuestas analizadas y teniendo en cuenta los niveles de progresión, se aprecia un cambio significativo, especialmente en lo referido a la tarea del historiador, seguido por la visión de la historia y los objetivos de esta. Sí que se han apreciado mayores dificultades en relación con la utilidad de la historia, si bien existe progresión, dado que los niveles iniciales eran más bajos. De esta manera, parece que la metodología basada en contenidos y competencias históricas puede favorecer el desarrollo del pensamiento histórico. Para comprender esos cambios, tenemos que tener en cuenta la importancia del trabajo con fuentes, la aplicación del método del historiador, el análisis de casos y la narración como herramientas didácticas, que favorecen el desarrollo de formas de pensamiento más complejas a través de la historia y la historiografia (Prieto Prieto et al., 2013). Como hemos visto, esto puede adaptarse a los diferentes niveles educativos y es compatible con la práctica en el aula de los docentes, así como con el currículo oficial.

Así pues, parece desprenderse de este estudio que la participación activa del alumnado y el planteamiento de actividades relacionadas con las competencias históricas favorece el aprendizaje y desarrollo del pensamiento histórico, si bien es un aspecto sobre el que habría que incidir en la práctica docente.

En cualquier caso, no hay que olvidar los límites de este trabajo, que se ha centrado en un grupo concreto. No obstante, pensamos que abre una puerta a posibles investigaciones futuras, teniendo en cuenta a un mayor número de alumnos y alumnas. Ahora bien, sí que debemos destacar la importancia de la investigación educativa en aras de servir a la práctica docente, teniendo como horizonte la mejora de la enseñanza y aprendizaje del alumnado. La didáctica de la historia debe responder a las nuevas demandas de la educación.

\section{Referencias}

Elliott, J. (2012). Haciendo Historia. Madrid: Taurus.

Fuentes, C. (2002). La visión de la historia por los adolescentes: revisión del estado de la cuestión en Estados Unidos y el Reino Unido. Enseñanza de las ciencias sociales: revista de investigación, 1, 55-68.

Fuentes, C. (2004). Concepciones de los alumnos sobre la historia. Enseñanza de las ciencias sociales: revista de investigación, 3, 75-83.

García Pérez, F. F. (2000). Los modelos didácticos como instrumento de análisis y de intervención en la realidad educativa. Biblio 3W. Revista Bibliográfica de Geografía y Ciencias Sociales, $207,1-15$. 
García Pérez, F. F. y De Alba, N. (2008). ¿Puede la escuela del siglo XXI educar a los ciudadanos y ciudadanas del siglo XXI? Scripta Nova: Revista electrónica de geografía y ciencias sociales, $12,270$.

García Pérez, F. F. y Porlán, R. (2000). El Proyecto IRES (Investigación y Renovación Escolar). Biblio 3W. Revista Bibliográfica de Geografia y Ciencias Sociales, 5(205), 1-16.

Gómez Carrasco, C. J. (2014). Pensamiento histórico y contenidos disciplinares en los libros de texto. Un análisis exploratorio de la edad Moderna en $2{ }^{\circ}$ de la ESO. Ensayos. Revista de la Facultad de Educación de la Universidad de Albacete, 29(1), 131-158.

Gómez Carrasco, C. J. y Chapman, A. (2017). Enfoques historiográficos y representaciones sociales en los libros de texto un estudio comparativo, España-Francia-Inglaterra. Historia y Memoria de la Educación, 6, 319-361. DOI: 10.5944/hme.6.2017.17132.

Gómez Carrasco, C. J. y Miralles Martínez, P. (2015). ¿Pensar históricamente o memorizar el pasado? La evaluación de los contenidos históricos en la educación obligatoria en España. Revista de estudios sociales, 52, 52-68. DOI: 10.7440/res52.2015.04.

Gómez Carrasco, C. J. y Miralles Martínez, P. (2017). Los espejos de Clío: usos y abusos de la historia en el ámbito escolar. Madrid: Sílex.

Gómez Carrasco, C. J., Miralles Martínez, P. y Molina Puche, S. (2015). Evaluación, competencias históricas y educación ciudadana. Revista de estudios sociales, 52, 9-13. DOI: 10.7440/res52.2015.01.

López Facal, R. (1999). Aprendizaje de la cultura y cultura del aprendizaje (reflexiones acerca de la enseñanza y el aprendizaje de la historia). Didáctica de las ciencias experimentales y sociales, 13, 33-54.

Martínez Rivera, C. A. y Martínez Rivera, V. G. (2012). El conocimiento escolar y las Hipótesis de Progresión: algunos fundamentos y desarrollos. Nodos y nudos: revista de la Red de Calificación de educadores, 4(32), 50-64. DOI: 10.17227/01224328.1799

Merchán Iglesias, F. J. (2001). El examen en la enseñanza de la historia. Didáctica de las ciencias experimentales y sociales, 15, 3-21.

Merchán Iglesias, F. J. (2005). Enseñanza, examen y control. Barcelona: Octaedro.

Merchán Iglesias, F. J. (2011). Práctica de la enseñanza y gobierno de la clase. Revista española de pedagogía, 69(250), 521-535.

Miralles Martínez, P., Molina Puche, S. y Monteagudo Fernández, J. (2017). Cuándo y cómo se evalúa en Geografía e Historia fases e instrumentos de evaluación del profesorado de ESO. Análisis de entrevistas y grupo de discusión en la Región de Murcia. Revista electrónica interuniversitaria de formación del profesorado, 20(1), 187-199. DOI: 10.6018/reifop.20.1.252821

Moreno Vera, J. R., Vera Muñoz, M. I. y Martínez Valcárcel, N. (2015). La invisibilidad en la enseñanza de la Historia: metodologías, estrategias y recursos. En A. M. Hernández, C. R. García y J. L. de la Montaña (coords.), Una enseñanza de las ciencias sociales para el futuro: recursos para trabajar la invisibilidad de personas, lugares y temáticas (pp. 925-931). Universidad de Extremadura, AUPDCS.

Navarro-Medina, E. y De-Alba-Fernández, N. (2011). El aprendizaje de la historia de España para la educación ciudadana. Investigación en la escuela, 75, 21-34.

Pagès, J. (2007). La enseñanza de las ciencias sociales y la educación para la ciudadanía en España. Didáctica geográfica, 9, 205-214.

Pérez-Guerrero, V. (2016). El lugar del examen en la Escuela: Estudio desde el campo de la Enseñanza de las Ciencias Sociales. Universidad de Sevilla.

Prieto Prieto, J. A., Gómez Carrasco, C. J. y Miralles Martínez, P. (2013). El uso de fuentes primarias en el aula y el desarrollo del pensamiento histórico y social. Una experiencia en Bachillerato. Clio: History and History Teaching, 39, 34-14. 
Sáiz Serrano, J. (2011). Actividades de libros de texto de Historia, competencias básicas y destrezas cognitivas, una difícil relación: análisis de manuales de $1^{\circ}$ y $2^{\circ}$ de ESO. Didáctica de las ciencias experimentales y sociales, 25, 37-64.

Santiesteban, A., y Pagès, J. (2010). La educación para la ciudadanía y la enseñanza de las ciencias sociales, la geografía y la historia. Iber: Didáctica de las ciencias sociales, geografía e historia, 64, 8-18.

Valls Montés, R., y López Facal, R. (2011). ¿Un nuevo paradigma para la enseñanza de la historia? Los problemas reales y las polémicas interesadas al respecto en España y en el contexto del mundo occidental. Enseñanza de las ciencias sociales: revista de investigación, 10, 75-86.

\section{CÓMO CITAR ESTE ARTÍCULO}

Fraile-Delgado, F. J. (2020). Pensar en Clío: la evolución de las concepciones historiográficas del alumnado de $1^{\circ}$ de Educación Secundaria Obligatoria. Didáctica de las ciencias experimentales y sociales, 39, 3-16. DOI: 10.7203/DCES.39.15512. 